\title{
75
}

\section{Exploring mathematics with image processing}

\author{
Steven L. Tanimoto \\ University of Washington \\ Seattle \\ USA
}

\begin{abstract}
Thanks to the ubiquity of powerful personal computers with high quality displays mathematics is being taught with a variety of exciting new visual approaches. One of these has particular interest in educating students of ages 12-20 where there is often a loss of interest in mathematics. Digital images are simultaneously visual and mathematical objects, and provide a natural vehicle for exploring arithmetic, number systems, functions, geometry, statistics and programming. A novel interactive learning environment is presented which supports a diverse group of student exercises, explorations and projects. This environment has been used on an experimental basis by a number of students in the Seattle area.
\end{abstract}

Main conference themes: methodologies

Educational areas: secondary education

Study topics: mathematics

Secondary keywords: graphics, interactive, learning systems, open learning, problem solving, user interface 


\section{INTRODUCTION}

During the early 1990s, the quality/cost ratio for PC graphics boards has increased significantly. Coupled with increased processing power of microprocessor CPUs the potential ability to do complex image processing has come to a wide user group. In the project Mathematics Experiences Through Image Processing (METIP) our research group at the University of Washington is investigating the use of image processing in middle school and high school mathematics.

The appeal of using digital images in mathematics education arises in part from the fact that a digital image is both a mathematical object and a visual object at the same time. An image, although made up of numbers in a twodimensional array, can represent almost anything in a student's personal world. This permits the possibility of a friendly, nonthreatening entry into a mathematical world for students of diverse backgrounds and interests. Related projects include the use of video to study dynamics [1] and still images to study scientific phenomena [2].

This paper describes some of the software and activities developed by the METIP project together with some of the rationale for using them.

\section{THE PIXEL CONNECTION}

Using images in mathematics requires a special kind of image display. Students must be able to see both the visual and mathematical aspects of the image. One of the first things they must learn is the relationship or connection between these two aspects of digital images. Consequently our 'Pixel Calculator' program provides a means to show digital images both as grey valued images and as arrays of integers. As the student uses a magnifier tool to zoom in on the image the pixel values appear numerically superimposed on the grey values when a certain level of magnification is reached.

To reinforce the connection between pixel values and degrees of brightness a means of manipulating pixel values is provided. The interface to the user appears as a pocket calculator. However, there is a special key labelled '\#' which represents the current value of a selected pixel. The Pixel Calculator interface can be seen in Figure 1.

The arithmetic operations of addition, subtraction, multiplication and division have novel applications in the Pixel Calculator. Addition can be used to lighten an image; subtraction darkens. Multiplication is used to boost the contrast, while division lowers contrast and darkens at the same time. Fine control of contrast can be obtained by using combinations of these operations. 


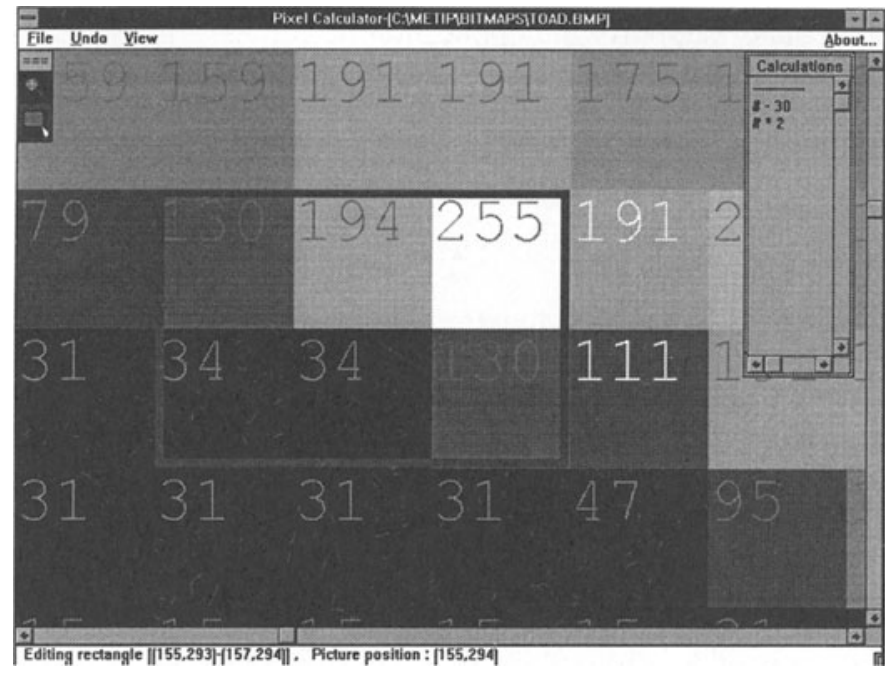

Fig. 1 Screen shot of the Pixel Calculator program

\section{Alternative number systems}

The Pixel Calculator program also provides an excellent example of the use of a number system different from those commonly taught in the past. Like clock arithmetic or other modulo $\mathrm{n}$ systems 'pixel arithmetic' uses a finite set of integers $0,1, \ldots, n-1$. However, here $n=256$, a power of 2 which establishes the context of computing. More interesting are the 'truncation semantics' associated with the arithmetic operations. For example, adding 1 to 255 in pixel arithmetic yields 255 , not 256 and also not 0 as one would get in a modular system. It is often surprising to teachers that division by zero is defined in pixel arithmetic, or that it is even possible for it to be defined. Pixel arithmetic is founded on a combination of practical aspects of image computing and mathematical consistency [3].

\section{Concept of function}

The METIP interactive learning environment supports students' understanding of the mathematical concept of function is several ways. First, each digital image itself represents a mapping from coordinate pairs to pixel values. A student may easily look up the pixel value for any coordinate pair by moving the mouse (and the magnifying tool) to the desired pixel and enlarging it. Several activities reinforce this notion of image as function including a game called Pixel Bingo and scavenger hunt searching challenges. 
The Pixel Calculator supports the application of certain arithmetic functions to selected sets of pixels. Whereas a standard pocket calculator operates on scalars the Pixel Calculator applies the specified operation simultaneously to all of the selected pixels. The use of the current pixel variable designated by '\#' allows the formulation of functions having a single independent variable, such as $f(\#)=255-\#$; this function computes the photographic negative of the selected portion of the image.

A third kind of mathematical function maps one image into another. Using a fast image transformation capability the METIP interactive learning environment allows a student to specify a mathematical formula which is then applied to the student's image. The students can interactively explore the effect of the formula on their image. In this context the image is a single mathematical object which is being manipulated by the function specified in the formula.

\section{Geometric transformations}

One class of functions which students find particularly interesting, are those which move, rotate, shrink, twist or distort whole images. The METIP interactive learning environment provides two methods of specifying geometric transformations. The first method works through the formulas which symbolically give the relationship between source image and destination image. The other method works through a direct manipulation geometric interface which provides control lines as 'handles' for shaping a geometric transformation.

The formulas which specify geometric transformations, may combine a variety of available functions and these may reference the image either in terms of Cartesian coordinates or in terms of polar coordinates. Figure 2 shows an original image and two transforms specified with formulas which manipulate the images using polar coordinates.

An illustration of an image distortion specified through the control line interface is shown in Figure 3. Although control lines provide a convenient and intuitive way for students to specify geometric distortions, it is also possible to specify the lines symbolically. This makes it possible to get fine control of the transformation as well as to study the effects of the transformation quantitatively.

\section{Convolution}

The Pixel Calculator and Image Warper programs are directed at students who may be new to the very idea of representing images numerically and processing them by computer. Another program we have developed, the 'Convolver,' is intended to assist students in exploring image processing with more sophisticated transformations. 


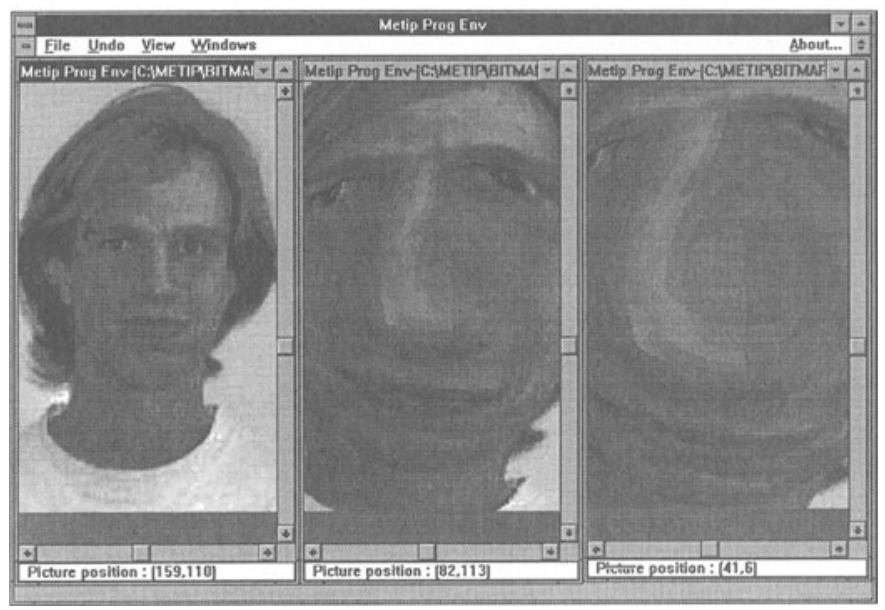

Fig. 2 (a) Original image of a student, (b) after transformation by

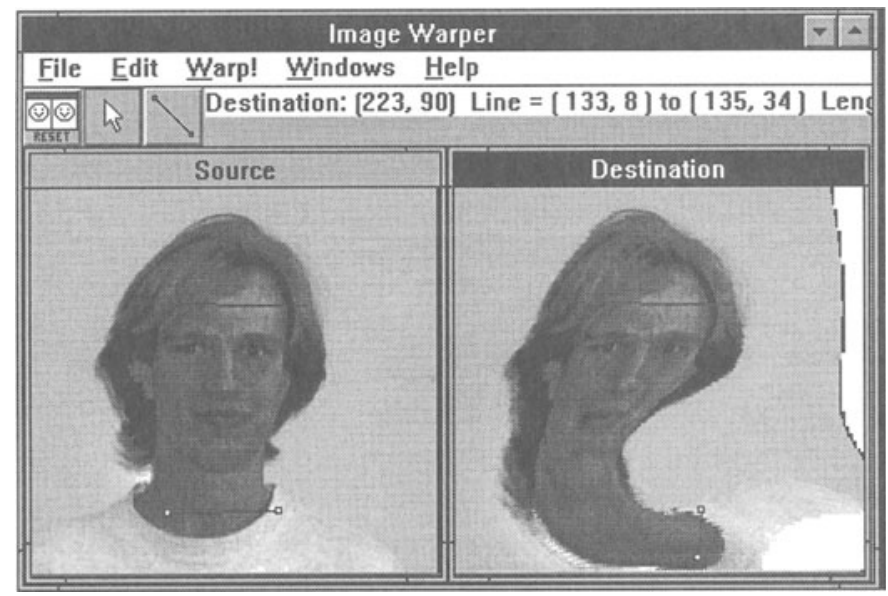

Fig. 3 Screen shot of the Image Warper

The fundamental difference in the sorts of processing done by the Pixel Calculator and the Convolver is that the Pixel Calculator operates on each pixel independently producing a new pixel value which is a function of only one original pixel value. The Convolver by contrast produces new pixel values which are weighted sums of several neighbouring pixel values of the original 
image. Combining two or more pixel values to produce a single new pixel value is a potentially much richer sort of image processing. Figure 4 illustrates the Convolver.

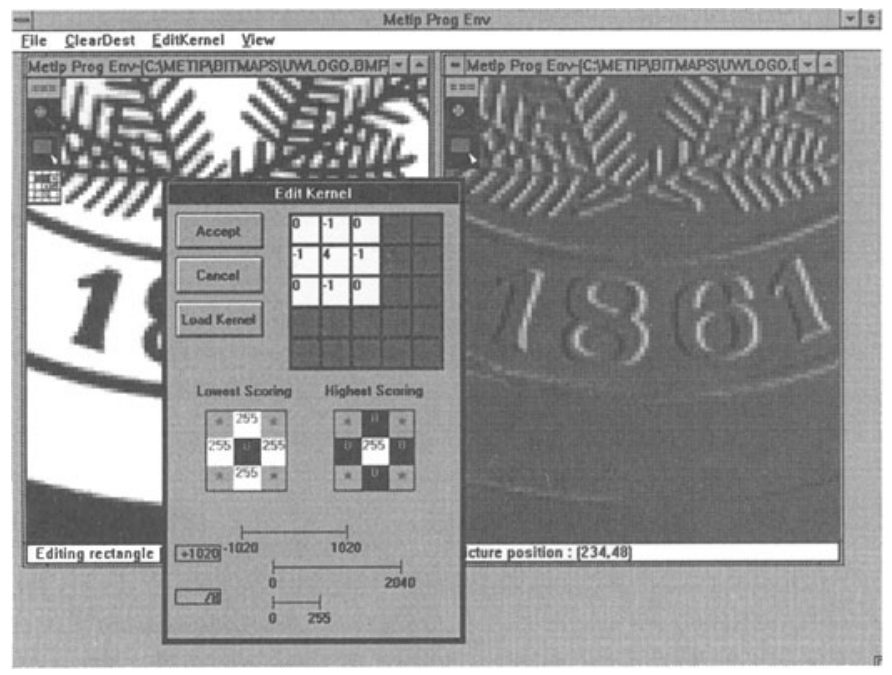

Fig. 4 Screen shot of the Convolver

The Convolver provides three different means of visualizing the convolution operation. First, it allows the student to paint with a convolving brush making it easy to see the computations involved in producing a single output pixel value. Second, it provides an animation mode in which the convolution process is applied slowly to all the pixels in any rectangular region selected by the student with each computation displayed. This animation also shows the raster scanning order of computation within the selected rectangle. The third means of showing convolution is full speed computation followed by the simultaneous display of all updated pixels. Although this method hides the individual pixel computations, it presents the overall visual changes to the image very clearly.

In addition to computing and displaying convolution operations the Convolver supports user editing of the kernel of convolution. Kernels of dimensions up to 5 by 5 are supported. The student does not have to worry about normalizing the convolved image to make its values displayable. An adaptive normalization mechanism is provided and the kernel editor updates a display of the normalization parameters whenever the kernel is altered. 
Learning activities for novice image processors which use the Convolver include:

- smoothing a blocky image to make a face easier to recognize;

- enhancing the edges in an image to give an embossing effect;

- detection of the corners of light and dark regions to illustrate a simple kind of robot vision.

\section{An applications programming interface}

Once the Pixel Calculator was developed we decided to repackage the user interface essentials in a way which might make it easier to develop additional image processing programs. The result was a piece of software which we call the METIP Programming Environment. This graphical user interface and library can be run independently or can be launched and commanded by a Windows application program written in $\mathrm{C}, \mathrm{C}++$ or another language. For example, it has been used with Visual Basic and with XLISP-STAT (a dialect of Lisp).

A possible use for the METIP Programming Environment which we have not yet had time to explore, is to support introductory programming with a visual approach complementary to that of Logo. Logo with its turtle graphics has a vector graphics based approach. A METIP introduction to programming would also be graphical, but based on manipulation of digital images (arrays of pixels). Although Logo provides students a controllable visual domain, METIP could permit students to work with visual objects which, through their depictions of friends and heroes, relate directly to the personal worlds of the students. This might help some of the more reluctant students to overcome their phobias or laziness and embrace computer programming as a powerful and expressive modality of communication.

\section{RELATION TO THE NCTM STANDARDS}

The National Council of Teachers of Mathematics in the United States published their Standards for Curriculum and Evaluation of School Mathematics in 1989. Since then the community of mathematics teachers has been struggling to bring everyday practice into conformity with the standards. Here are three ways in which METIP activities can assist teachers in meeting the standards.

\section{Modelling}

By using mathematical representations of natural phenomena such as images students can not only appreciate the relevance of mathematics to practical concerns, but they can also develop their own intuition about appropriate 
modelling methods. The Pixel Calculator provides a simple tool for understanding images as mathematical objects. The issue of resolution in modelling is more obvious in the case of images than in anything else. Resolution, however, is fundamental in digital representation of sound, in weather prediction, in computer-aided design of airplane wings and in many other domains.

\section{Connections}

A subject like mathematics must be made relevant to students by making its applications explicit, connecting its ideas to those of other subjects and putting mathematical activities into broader contexts. Image processing has many connections to nonmathematical subjects; for example, biological images from microscopes can be enhanced by image processing. Within mathematics many concepts such as area, perimeter, proportion, angle and number theory can be discussed in terms of digital images; for instance, with the Pixel Calculator one can zoom in on part of an image and determine area of an object by counting the pixels in the object.

\section{Problem formulation}

Whereas traditionally students have been assigned problems to solve the NCTM standards encourage teachers to nurture students' ability to pose problems as well. When a learning environment supports learning by discovery, it helps create opportunities for problem posing [5]. The Pixel Calculator, Image Warper and METIP Programming Environment provide an interactive learning environment which empowers students to pursue open ended activities in which they evaluate the effects of transformations on images, devise strategies to achieve particular effects and pose, as well as solve problems.

There are many other aspects to the NCTM standards including communication, curricular content, estimation, etc., but space does not permit a full discussion of all these and their relationships to image processing.

\section{CONCLUSIONS}

We have found that students are eager to work with digital image processing. They take particular pleasure in transforming images of themselves, friends and teachers. On one occasion we used a digital camera to take photos of the students in small groups. Before the next class we loaded up the hard drives with different subsets of these images. When the students came in and discovered the images, they became very excited, ran from one computer to 
another to see what images were there and the class got out of control; the activity had to be altered.

Our materials have been designed primarily to introduce students to image processing in such a way as to raise their motivation to learn mathematics. Evaluation of the materials is continuing and we expect to report on the results in the future. While pursuing our main goal, we found two other needs which our materials easily satisfy. One of these is students' need to know at a very elementary level what image processing is, independently of its relationship to mathematics. The other is the need of students to work with computers doing something other than playing video games or typing with a word processing program.

Image processing can enrich the K-12 (secondary) mathematics curriculum in a variety of ways consistent with the standards set forth by the NCTM. Image processing can be considered new, relevant content. And therefore it should be taught, regardless of whether it helps children learn arithmetic, algebra or probability. Because of its connections to nonmathematical subjects it can potentially be integrated with not only mathematics, but also art, science and English (in a context of multimedia authoring perhaps).

Evaluation of these materials is in progress, and we anticipate learning about how these image processing materials impact motivation and achievement in different educational settings.

\section{ON-LINE INFORMATION}

Additional information about Mathematics Experiences Through Image Processing is available in the World Wide Web at the URL (Uniform Resource Locator): http://www.cs.washington.edu/research/metip/

\section{ACKNOWLEDGEMENTS}

The author would like to thank L. Bricker, S. Fain, P. Goldenberg, J. King, A. Kullavanijaya, M. LeBrassuer, D. Lee, E. McLain, S. Monk, A. Rothenberg and the National Science Foundation for their contributions to those aspects of the METIP project described here. The project has been supported in part by NSF Grant No. MDR-9155709. 
The fundamental difference in the sorts of processing done by the Pixel Calculator and the Convolver is that the Pixel Calculator operates on each pixel independently producing a new pixel value which is a function of only one original pixel value. The Convolver by contrast produces new pixel values which are weighted sums of several neighbouring pixel values of the original image. Combining two or more pixel values to produce a single new pixel value is a potentially much richer sort of image processing. Figure 4 illustrates the Convolver.

\section{REFERENCES}

1. Rubin, A. (1993) Video laboratories: Tools for scientific investigation. Communications of the Assoc. for Computing Machinery, 36 (5) pp. 64-5.

2. Garrison, A. (1990) Seeing is learning. MacWorld, December, p. 151.

3. Tanimoto, S. L., King, J. R., and Lee, D. (1993) Pixel arithmetic in mathematics education through image processing. Technical Report 93-05-08, Department of Computer Science and Engineering, University of Washington. Seattle WA.

4. National Council of Teachers of Mathematics, (1989) Curriculum and Evaluation Standards for School Mathematics, Reston, VA: NCTM.

5. Yerushalmy, M. and Chazan, D. (1987) Effective problem posing in an inquiry environment: a case study using the Geometric Supposer. Proc. Eleventh International Conference on Psychology of Mathematics Education, 2, pp. 53-60. 